\title{
Díadas de cuidado y competencia para cuidar al alta hospitalaria en un hospital de Bogotá
}

\author{
Jenny C Rojas-Barbosa', Mónica P Quemba-Mesa²
}

\begin{abstract}
RESUMEN
Introducción. Con frecuencia las personas con enfermedades crónicas requieren un cuidado especial en su hogar, siendo en este contexto fundamental la competencia de cuidado.

Objetivo. Identificar las características y asociaciones del cuidado informal y de la competencia para cuidar de la díada cuidador-persona con enfermedad crónica, durante el alta hospitalaria en un hospital de alta complejidad de Bogotá - 2016.

Metodología. Estudio cuantitativo-descriptivo, transversal-analítico; en la medición se usó la encuesta de caracterización de la díada: cuidador-persona con enfermedad crónica, y el instrumento de competencia de cuidado en el hogar, versión abreviada CUIDAR.

Resultados. Participaron 97 díadas. Los cuidadores eran, en su mayoría, mujeres (79,4\%) con edad promedio de 43,7 $( \pm 15,5)$ años, con una trayectoria como cuidadores en promedio de 4,3 años. Las personas con enfermedad crónica eran mayormente mujeres $(67,0 \%)$, con edad promedio de 64,3 años, y con antecedentes patológicos de diabetes mellitus $(55,7 \%)$ e hipertensión arterial $(48,5 \%)$. Cuidadores perciben sentir carga $(61,9 \%)$ y pacientes ser una carga $(57,7 \%)$; las díadas perciben mínima satisfacción con el apoyo recibido y están más familiarizadas con el uso del teléfono y televisión. La competencia para cuidar fue valorada como alta por el cuidador (44,8 puntos promedio). Dimensiones de la competencia de cuidado se asociaron $(p<0,05)$ con escolaridad, compromiso religioso, carga percibida, edad del paciente, entre otros aspectos.

Conclusiones. Las díadas de cuidado cuentan con características relacionadas con el nivel de competencia para el cuidado, estas son de importancia en la estructuración de intervenciones para mejorar el bienestar de los cuidadores. Palabras clave: enfermedad crónica; cuidadores; alta del paciente; atención domiciliaria de salud.
\end{abstract}

${ }^{1}$ Fundación Santafe de Bogotá. Bogotá. Colombia

2 Universidad de Boyacá. Tunja. Boyacá. Colombia

Correspondencia: Mónica Paola Quemba Mesa. Dirección: Calle 73 \# 2H-22 apto 401 Tunja, Colombia, Teléfono 3138501108.

Correo electrónico: mpquemba@uniboyaca.edu.co

Citar este artículo así:

Rojas Barbosa JC, Quemba Mesa MP. Díadas de cuidado y competencia para cuidar al alta hospitalaria en un hospital de Bogotá. Revista Inves-tig Salud Univ Boyacá. 2020;7(1): 71-86. doi: https://doi.org/10.24267/23897325.417 


\title{
Dayds of care and competition to care for hospital discharge in a Bogotá hospital
}

\begin{abstract}
Introduction. People with chronic diseases often require special care in their home, being in this fundamental context the competence of care.

Objective. Identify the characteristics and associations of informal care and competition to take care of the "Caregiver-Person" dyads with chronic illness during hospital discharge in a high complexity hospital in Bogotá - 2016.

Methodology. Quantitative - descriptive, analytical cross-sectional study, using the "Characterization survey of the Caregiver-Person dyad with chronic disease" and the instrument of "Home care competence - abbreviated version CUIDAR-"

Results. 97 days participated. Caregivers were mostly women $(79,4 \%)$ with an average age of 43,7 years old and have been caregivers in average 4,3 years. The people with chronic disease were mostly women $(67,0 \%)$ with an average age of 64,3 years old and with a pathological history of diabetes mellitus $(55,7 \%)$ and high blood pressure $(48,5 \%)$. Caregivers perceive feeling a burden $(61,9 \%)$ and patients being a burden $(57,7 \%)$, dyads perceive minimal satisfaction with the support received, and they are more familiar with the use of phone and television. The competence to care was rated as high by the caregiver $(44,8$ points average). Dimensions of care competence were associated $(p<0,05)$ with schooling, religious commitment, perceived burden, patient age, among other aspects.
\end{abstract}

Conclusions. Caregiver-Person dyads have characteristics related to the level of competence for care, these are being of importance in structuring interventions to improve the well-being of caregivers.

Keywords. chronic disease; caregivers; patient discharge; home nursing. 


\title{
Diades de cuidado e competência para cuidar da alta hospitalar em um hospital de Bogotá
}

\begin{abstract}
RESUMO
Introdução. As pessoas com doenças crônicas frequentemente necessitam de cuidados especiais em seu domicílio, sendo neste contexto fundamental a competência de cuidar.

Objetivo. Identificar as características e associações de atenção informal e competição para cuidar das díades "Cuidador-Pessoa" com doença crônica durante a alta hospitalar em um hospital de alta complexidade em Bogotá - 2016.

Metodologia. Estudo quantitativo - descritivo, analítico, transversal, utilizando o "Levantamento de caracterização da díade Cuidador-Pessoa com doença crônica" e o instrumento "Competência em assistência domiciliar - versão abreviada CUIDAR-"

Resultados. 97 dias participaram. Os cuidadores eram em sua maioria mulheres $(79,4 \%)$, com idade média de 43,7 anos e cuidadores em média 4,3 anos. As pessoas com doença crônica eram majoritariamente mulheres $(67,0 \%)$, com idade média de 64,3 anos e história patológica de diabetes mellitus $(55,7 \%)$ e hipertensão arterial $(48,5 \%)$. Os cuidadores percebem sentir um fardo $(61,9 \%)$ e os pacientes um fardo $(57,7 \%)$, as díades percebem uma satisfação mínima com o apoio recebido e estão mais familiarizados com o uso de telefone e televisão. A competência para cuidar foi classificada como alta pelo cuidador (44,8 pontos em média). As dimensões da competência assistencial foram associadas ( $p$ $<0,05)$ à escolaridade, comprometimento religioso, carga percebida, idade do paciente, entre outros aspectos.
\end{abstract}

Conclusões. os dias de atendimento têm características relacionadas ao nível de competência para o cuidado, sendo de importância na estruturação de intervenções para melhorar o bem-estar dos cuidadores.

Palavras-chave: doença crônica; cuidadores; alta do paciente; assistência médica domiciliar. 


\section{INTRODUCCIÓN}

Las personas con Enfermedades Crónicas no Transmisibles (ECNT) requieren, por la evolución y pronóstico de este tipo de patologías, un cuidado especial que va mucho más allá del escenario hospitalario; en este sentido, es de vital importancia tanto el proceso de alta hospitalaria como el rol del cuidador en este contexto, pues el egreso al hogar implica, para el cuidador y para el paciente, la toma de decisiones sobre cuándo y cómo comenzar o terminar las actividades de cuidado, como: modificar medicamentos, iniciar planes de emergencia, adoptar las rutinas o buscar la ayuda profesional (1).

El estudio de la competencia de cuidado del cuidador familiar, es decir, "la habilidad que se desarrolla y se constituye en aspectos positivos de la experiencia del cuidado de una persona con enfermedad crónica" (2), se hace fundamental como medida para evaluar integralmente las capacidades, habilidades y actitudes para el cuidado en el hogar de quien ejerce el rol de cuidador, ya que el cuidado de otra persona como proceso terapéutico e interpersonal, es dinámico y exige responsabilidad y compromiso en el actuar (3).

El cuidado que brindan los cuidadores familiares se encuentra enmarcado en un contexto complejo, en el cual se aumenta tanto de la esperanza de vida como de la prevalencia de las ECNT. De acuerdo con la Organización Mundial de la Salud (OMS), se evidencia que las ECNT están creciendo de forma sostenida en numerosos países de ingresos bajos y medios, hecho que se relaciona con la transición sociodemográfica en la que se estiman el crecimiento en la esperanza de vida, con un promedio para el 2030 de entre 70 y 80 años; el decremento en la natalidad; unas pautas de consumo y cambios en los modos de vida; la urbanización, y la comercialización mundial (4).

En Colombia, la hipertensión y la diabetes mellitus son las principales enfermedades precursoras de ECNT como la insuficiencia renal, en población cada vez más senil (5). Para el periodo 2014, se reportaron por entidades administradoras del régimen contributivo un $67,84 \%$ de personas diagnosticadas con hipertensión arterial, un $29,80 \%$ de afiliados al régimen subsidiado con este diagnóstico, y un 2,36\% fueron reportados por Fuerzas Militares y Policía Nacional (5). En el caso de la Diabetes Mellitus, se encontró un $74,35 \%$ de personas diagnosticadas y afiliadas al régimen contributivo, un $22,88 \%$ afiliadas al régimen subsidiado y un $2,77 \%$ afiliados al régimen de especial (5).

El aumento de los adultos mayores con ECNT presenta un panorama importante desde el punto de vista de la salud pública, pues es frecuente encontrar que ellos viven con una o varias enfermedades crónicas, las cuales se acompañan de 
discapacidad física, psíquica, sensorial y mental; todo lo anterior, lleva a la persona a tener algún grado de dependencia en las actividades de la vida diaria (6). En este sentido, dichos adultos mayores pueden ser, parcial o totalmente, dependientes en las actividades de la vida diaria; además, requieren cambios en su estilo de vida, los cuales deben ser progresivos, ya que traen consigo necesidades y procesos de adaptación (7). En este contexto, se hace necesario el apoyo del cuidador familiar quien, por lo general, es una persona cercana o con grado de parentesco que, de acuerdo con los estudios regionales, tiene las siguientes características: sexo femenino en un $87.8 \%$ (madre, esposa o hija), rango de edad entre 27 y 83 años, y alto riesgo de desarrollar el síndrome del cuidador (problemas físicos como algias musculares, cansancio, dolor de espalda y cefalea; además de la aparición de problemas psicológicos como irritabilidad, ansiedad y depresión) (6).

Diversos trabajos escritos justifican la importancia de estudiar la competencia del cuidador familiar del paciente con ECNT; adicionalmente, constituyen un valioso aporte al conocimiento de las necesidades de cuidado, en escenarios extra hospitalarios como el hogar. Así mismo, teniendo en cuenta las dinámicas en salud actuales, con estancias hospitalarias más cortas y la asignación de responsabilidades de autogestión y manejo al paciente y su familia en el hogar, describir el fenómeno de la competencia del cuidador familiar cobra un valor importante en el ámbito de la investigación clínica que orientará en la generación de intervenciones interdisciplinarias integrales, acordes con estas realidades.

Se hace necesario que el personal de salud determine que tan preparado y dispuesto está el cuidador, aspecto que se puede establecer a través del análisis de la competencia de cuidado del cuidador, herramienta integral que media de forma positiva y brinda estabilidad en la relación de cuidado de la díada (8). Por lo anterior, este estudio se realiza con el objetivo de identificar las características y asociaciones del cuidado informal y de la competencia para cuidar la díada cuidador-persona con enfermedad crónica durante el alta hospitalaria en un hospital de alta complejidad de Bogotá - 2016.

\section{METODOLOGÍA}

Estudio cuantitativo-descriptivo, de corte transversal analítico. La población estuvo conformada por las díadas de cuidado-paciente con ECNT hospitalizado y su cuidador, de una institución hospitalaria de alta complejidad de la ciudad de Bogotá, durante el año 2016. La muestra mínima requerida fue de 97 díadas de cuidado, calculada con el programa Epidat 3.1, a partir de una población de 8.927 pacientes egresados al año, en una proporción esperada de $28,8 \%$ de cuidadores de alto nivel de competencia para el cuidado 
(9), con una confianza del 95\% y una precisión del 0.09, esto siguiendo la fórmula para proporción conociendo el tamaño de la población:

$$
n=\frac{N \times Z_{a}{ }^{2} \times p \times q}{d^{2} \times(N-1)+Z_{a}{ }^{2} \times p \times q}
$$

El muestreo se realizó por conveniencia, seleccionando a las díadas de cuidado que aceptaran su participación y que cumplieran con criterios de inclusión como: estar conformadas por el cuidador principal y un paciente diagnosticado con ECNT, hospitalizado en la institución participante y en proceso de alta hospitalaria, ambos mayores de 18 años y con algún grado de parentesco o de cercanía entre ellos; por otra parte, como parte de los criterios de exclusión se contemplaron las díadas de cuidado en las que el cuidador tenía algún grado de discapacidad física, déficit cognitivo o de comunicación.

Como parte de los instrumentos de recolección de datos usados está la encuesta de caracterización de la díada cuidador-persona con enfermedad crónica, instrumento en el que se identifican diversas variables, agrupadas en tres dimensiones: identificación de condiciones del paciente y su perfil sociodemográfico, percepción de carga y de apoyo, y medios de información y comunicación. Sobre esa encuesta se reporta para el contexto local una validez de expertos que califica el $100 \%$ de los ítems como claros, coherentes, sufi- cientes, relevantes y esenciales para cada una de las dimensiones (10).

También se usó el instrumento de "Competencia de cuidado en el hogar - versión abreviada del cuidador familiar de persona con enfermedad crónica - CUIDAR-", el cual cuenta con 20 ítems agrupados en cinco dimensiones: C: Conocimiento; U: Unicidad (condiciones personales); I: Instrumental y procedimental; D: Disfrutar (bienestar); A: Anticipar (predictiva); y R: Relación social e interacción. En relación con la validez, su consistencia interna fue reportada con un buen rendimiento con un alfa de Cronbach de 0.96 (11).

Antes de iniciar la recolección de datos se realizó una prueba piloto con 5 cuidadores, la cual permitió afinar el proceso de aplicación de los instrumentos en campo. En relación con las consideraciones éticas se contó con aval del Comité de Ética en Investigación de las instituciones que participaron, se aplicó el proceso de consentimiento informado y se siguieron las pautas que en la materia da la normatividad nacional e internacional, según las cuales, este es un estudio sin riesgo de acuerdo con la Resolución 8430 de 1993 del Ministerio de Salud de Colombia.

Para el análisis de datos se construyó una matriz de tabulación en Microsoft Excel y su análisis estadístico se hizo en el programa estadístico SPSS versión 24.0; inicialmente, se consideró 
un análisis univariado utilizando para tal fin frecuencias absolutas y relativas en variables cualitativas, y en variables cuantitativas medidas de tendencia central y de dispersión. En el análisis bivariado para determinar si existe o no diferencia entre variables de interés se usarán pruebas como Chi-cuadrado de Pearson y la prueba de la Mediana, esto dependiendo del tipo de variables y de si su comportamiento guarda una distribución normal; por otra parte, para la interpretación de estas pruebas se asume un valor $p$ de significancia estadística de menor a 0,05.

\section{RESULTADOS}

Se contó con la participación de 97 díadas de cuidado que cumplieron con los criterios de selección establecidos. Así, los pacientes con ECNT se caracterizaron por ser, en su mayoría, de género femenino $(67,0 \%)$, conservar su independencia $(88,7 \%)$ y sus capacidades cognitivas $(94,8 \%)$, tener como antecedentes patológicos DM $(55,7 \%)$ y HTA $(48,5 \%)$, presentar bajos niveles de escolaridad $(72,2 \%)$, y mostrar un nivel medio de compromiso religioso $(64,9 \%)$, entre otras características. Su edad promedio es de 64,3 años, tienen en promedio 3,4 diagnósticos médicos y el promedio de tiempo desde el diagnóstico de la ECNT es de 5,5 años (ver tabla No. 1).

De igual manera, los cuidadores se caracterizan por ser, en su mayoría, de género femenino
$(79,4 \%)$, conservar su independencia $(99,0 \%)$ y sus capacidades cognitivas (100\%), tener niveles medios de escolaridad $(45,4 \%)$, ser de estratos $1(45,4 \%)$ y $2(46,4)$, y mostrar un nivel medio de compromiso religioso $(72,2 \%)$, entre otras características. Su edad promedio es de 43,7 años, tienen en promedio 0,3 diagnósticos médicos (lo que equivale el $33 \%$ de cuidadores diagnosticados) y llevan como cuidadores aproximadamente 4,3 años (ver tabla No. 1).

Tabla 1. Identificación de condiciones del perfil sociodemográfico de las díadas de cuidado

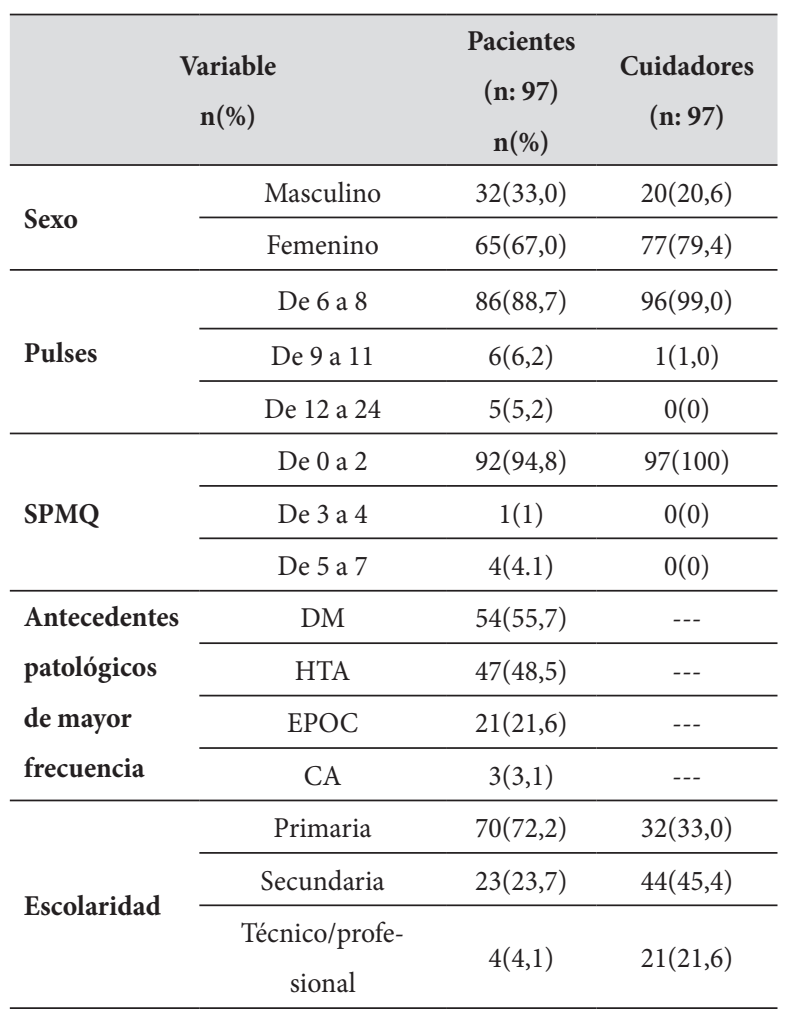




\begin{tabular}{|c|c|c|c|}
\hline \multicolumn{2}{|c|}{$\begin{array}{c}\text { Variable } \\
\mathbf{n}(\%)\end{array}$} & $\begin{array}{c}\text { Pacientes } \\
\text { (n: } 97) \\
\text { n(\%) }\end{array}$ & $\begin{array}{c}\text { Cuidadores } \\
\text { (n: 97) }\end{array}$ \\
\hline \multirow{2}{*}{ Procedencia } & Urbana & $92(94,8)$ & $92(94,8)$ \\
\hline & Rural & $5(5,2)$ & $5(5,2)$ \\
\hline \multirow{5}{*}{ Estado civil } & Soltero & $27(27,8)$ & $30(30,9)$ \\
\hline & Casado & $22(22,7)$ & $31(32,0)$ \\
\hline & Separado & $10(10,3)$ & $17(17,5)$ \\
\hline & Viudo & $31(32,0)$ & $6(6,2)$ \\
\hline & Unión libre & $7(7,2)$ & $13(13,4)$ \\
\hline \multirow{4}{*}{ Ocupación } & Hogar & $78(80,4)$ & $37(38,1)$ \\
\hline & Empleado & $6(6,2)$ & $34(35,1)$ \\
\hline & Independiente & $10(10,3)$ & $24(24,7)$ \\
\hline & Estudiante & $3(3,1)$ & $2(2,1)$ \\
\hline \multirow{3}{*}{$\begin{array}{l}\text { Estrato so- } \\
\text { cioeconómico }\end{array}$} & 1 & $50(51,5)$ & $44(45,4)$ \\
\hline & 2 & $40(41,2)$ & $45(46,4)$ \\
\hline & 3 & $7(7,2)$ & $7(7,2)$ \\
\hline \multirow{3}{*}{$\begin{array}{l}\text { Nivel de } \\
\text { compromiso } \\
\text { religioso }\end{array}$} & Bajo & $9(9,3)$ & $6(6,2)$ \\
\hline & Medio & $63(64,9)$ & $70(72,2)$ \\
\hline & Alto & $25(25,8)$ & $21(21,6)$ \\
\hline \multirow{4}{*}{\multicolumn{2}{|c|}{ Variable }} & Pacientes & Cuidadores \\
\hline & & $\mathrm{X} \pm \mathrm{DE} /$ & $\mathrm{X} \pm \mathrm{DE} /$ \\
\hline & & Mínimo - & Mínimo - \\
\hline & & Máximo & Máximo \\
\hline \multirow{2}{*}{\multicolumn{2}{|c|}{ Edad }} & $63,4 \pm 19,17$ & $43,7 \pm 15,5 /$ \\
\hline & & / 19-96 & $17-77$ \\
\hline \multirow{2}{*}{\multicolumn{2}{|c|}{ Número de diagnósticos médicos }} & $3,4 \pm 2,3 /$ & $0.3+07 / 0-4$ \\
\hline & & $1-10$ & ב \\
\hline \multirow{2}{*}{\multicolumn{2}{|c|}{ Tiempo de diagnóstico de ECNT }} & $5,5 \pm 5,04 /$ & \\
\hline & & $1-25$ & \\
\hline
\end{tabular}

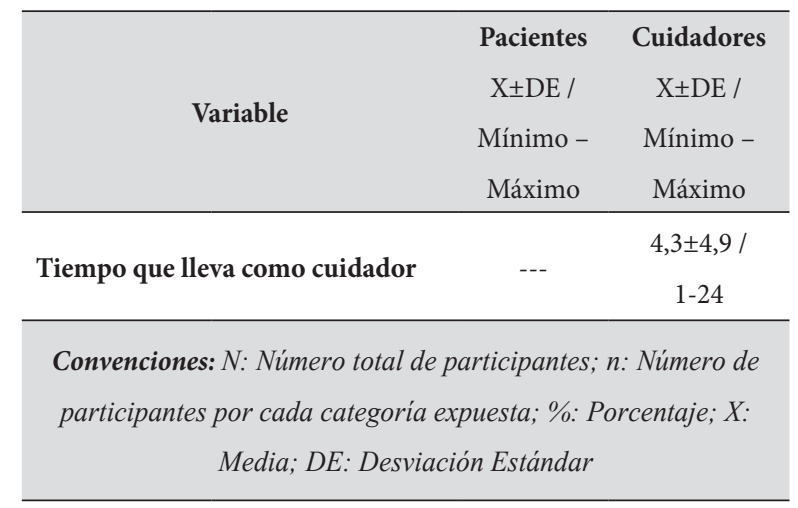

Fuente: la autora, procesamiento estadístico de los datos

En relación con la percepción de carga y de apoyo de las díadas de cuidado, se evidencia que la mayoría reporta un único cuidador $(68,0 \%)$, y que tanto el paciente como el cuidador perciben una mínima satisfacción con el apoyo psicológico $(95,9 \%)$, familiar $(99 \%$ y $95,9 \%)$, religioso $(74,2 \%$ y $87,6 \%)$, económico $(99 \%)$ y social $(96,9)$ con el que cuentan; en su mayoría, los cuidadores son hijos(as) de los pacientes $(63,9 \%)$ y no tuvieron experiencias previas como cuidadores (82,5\%). Asimismo, la percepción del paciente de ser una carga es muy alta $(57,7 \%)$, mientras que la percepción del cuidador de sentirse cargado es alta $(61,9 \%)$. Con respecto a la percepción que los pacientes tienen sobre su bienestar, la mayoría lo califica entre ninguno y mínimo en la dimensión física (61,9\%), psicológica $(67,0 \%)$, social $(62,9 \%)$ y espiritual $(56,7 \%)$; en contraste, la percepción que los cuidadores tienen sobre su bienestar es valorada, por la mayoría, en los dos niveles más 
altos en la dimensión física (55,6\%), psicológica $(54,7 \%)$, social $(61,9 \%)$ y espiritual $(65,0 \%)$. De igual manera, hay diferencia entre el reporte del promedio de número de horas diarias por el paciente (18 h) y por el cuidador (15 h).

Frente a la autovaloración que las díadas de cuidado hacen del uso de los medios de comunicación e información, tanto los pacientes como los cuidadores, en su mayoría, refieren el uso del teléfono, reportando un alto conocimiento $(67,0 \%-90,7 \%)$, acceso $(64,9 \%-88,7 \%)$ y frecuencia de uso $(60,8 \%-85,9 \%)$; igualmente, en lo concerniente al uso de la televisión, reportan también un alto conocimiento $(75,3 \%-93,9 \%)$, acceso $(59,8 \%-85,6 \%)$ y frecuencia de uso $(47,4 \%-63,9 \%)$; sin embargo, el uso de otros medios como la radio, el computador y el internet fueron calificados, en general, con un nivel bajo en los ítems: conocimiento, acceso y frecuencia de uso. De igual manera, la mayoría de pacientes y cuidadores expresa no haber tenido uso previo de TIC's en el cuidado (97,9\% y 92,8\%) y bajo el nivel de apoyo percibido por medio de las TIC's (96,9\% para ambos). Asimismo, las díadas están de acuerdo en el posible orden de uso de preferencia que tendrían de las TIC's en mención, siendo estas, de mayor a menor, la televisión, el teléfono, la radio, el computador y el internet.

Del mismo modo, la competencia para el cuidado del cuidador familiar antes del alta hospitalaria, en general, fue valorada como alta al obtener en promedio 44,8 puntos sobre el valor máximo posible (60 puntos); asimismo, las siguientes dimensiones también fueron valoradas con puntuaciones promedio altas: Conocimiento $(7,5$ puntos sobre 9 posibles), Unicidad (8,3 puntos sobre 12 posibles), Instrumental y procedimental (8,7 puntos sobre 9 posibles), Anticipación (5,7 puntos sobre 6 posibles) y Relación social e interacción ( 8,7 puntos sobre 12 posibles) (ver tabla No. 2). La dimensión que fue valorada con una puntuación promedio más baja fue Disfrutar, esta quedó en un nivel medio (5,8 puntos sobre 12 posibles) (ver tabla No. 2).

Por otro lado, el análisis bivariado identificó, por medio de las pruebas Chi-cuadrado de Pearson (tomando únicamente los valores significativos $y$ en los cuales en las tablas de $2 \times 2$ se contaba con frecuencias frecuencias superiores a 5) y la Prueba de la Mediana, las relaciones significativas de las variables de interés con la Competencia para el cuidado en cada una de sus dimensiones, evidenciando que entre los aspectos con mayor relevancia se encuentran el nivel de dependencia, el compromiso religioso, la carga percibida, la edad, entre otras (ver tabla No. 3). 
Tabla 2. Competencia para el cuidado - versión abreviada para el cuidador

\begin{tabular}{|c|c|c|c|c|c|c|}
\hline \multirow{2}{*}{$\begin{array}{l}\text { Îtem } \\
\text { n(\%) }\end{array}$} & & $\begin{array}{c}\text { Casi nunca o } \\
\text { nunca }\end{array}$ & Pocas veces & Con frecuencia & $\begin{array}{c}\text { Casi siempre o } \\
\text { siempre }\end{array}$ & \multirow{2}{*}{$\begin{array}{c}\text { Puntuación } \\
\text { promedio }\end{array}$} \\
\hline & & $\mathrm{n}(\%)$ & $\mathrm{n}(\%)$ & $\mathrm{n}(\%)$ & & \\
\hline \multirow{3}{*}{ C: Conocimiento } & 1 & $1(1,0)$ & $15(15,5)$ & $11(11,3)$ & $70(72,2)$ & \multirow{3}{*}{$\begin{array}{l}7,5 / 9 \\
\text { Alta }\end{array}$} \\
\hline & 2 & $1(1,0)$ & $17(17,5)$ & $12(12,4)$ & $67(69,1)$ & \\
\hline & 3 & $1(1,0)$ & $17(17,5)$ & $11(11,3)$ & $68(70,1)$ & \\
\hline \multirow{4}{*}{$\begin{array}{l}\text { U: Unicidad (condiciones } \\
\text { personales) }\end{array}$} & 4 & $1(1,0)$ & $26(26,8)$ & $19(19,6)$ & $51(52,6)$ & \multirow{4}{*}{$\begin{array}{c}8,3 / 12 \\
\text { Alta }\end{array}$} \\
\hline & 5 & $1(1,0)$ & $32(33,0)$ & $30(30,9)$ & $34(35,1)$ & \\
\hline & 6 & $2(2,1)$ & $34(35,1)$ & $26(26,8)$ & $35(36,1)$ & \\
\hline & 7 & $2(2,1)$ & $32(33,0)$ & $21(21,6)$ & $42(43,3)$ & \\
\hline \multirow{3}{*}{$\begin{array}{l}\text { I: Instrumental y procedi- } \\
\text { mental }\end{array}$} & 8 & $1(1,0)$ & $0(0)$ & $7(7,2)$ & $89(91,8)$ & \multirow{3}{*}{$\begin{array}{c}8,7 / 9 \\
\text { Alta }\end{array}$} \\
\hline & 9 & $3(3,1)$ & $0(0)$ & $5(5,2)$ & $89(91,8)$ & \\
\hline & 10 & $0(0)$ & $0(0)$ & $6(6,2)$ & $91(93,8)$ & \\
\hline \multirow{4}{*}{ D: Disfrutar (bienestar) } & 11 & $7(7,2)$ & $40(41,2)$ & $26(26,8)$ & $24(24,7)$ & \multirow{4}{*}{$\begin{array}{c}5,8 / 12 \\
\text { Media }\end{array}$} \\
\hline & 12 & $16(16,5)$ & $47(48,5)$ & $16(16,5)$ & $18(18,6)$ & \\
\hline & 13 & $14(14,4)$ & $49(50,5)$ & $17(17,5)$ & $17(17,5)$ & \\
\hline & 14 & $13(13,4)$ & $54(55,7)$ & $15(15,5)$ & $15(15,5)$ & \\
\hline \multirow{2}{*}{ A: Anticipación (predictiva) } & 15 & $1(1,0)$ & $1(1,0)$ & $10(10,3)$ & $85(87,6)$ & \multirow{2}{*}{$\begin{array}{c}5,7 / 6 \\
\text { Alta } \\
\end{array}$} \\
\hline & 16 & $1(1,0)$ & $1(1,0)$ & $11(11,3)$ & $84(86,6)$ & \\
\hline \multirow{4}{*}{$\begin{array}{l}\text { R: Relación social e inte- } \\
\text { racción }\end{array}$} & 17 & $24(24,7)$ & $18(18,6)$ & $16(16,5)$ & $39(40,2)$ & \multirow{4}{*}{$\begin{array}{c}8,7 / 12 \\
\text { Alta }\end{array}$} \\
\hline & 18 & $4(4,1)$ & $16(16,5)$ & $33(34,0)$ & $44(45,4)$ & \\
\hline & 19 & $2(2,1)$ & $15(15,5)$ & $30(30,9)$ & $50(51,5)$ & \\
\hline & 20 & $2(2,1)$ & $9(9,3)$ & $26(26,8)$ & $60(61,9)$ & \\
\hline \multicolumn{6}{|c|}{ Convenciones: $n$ : Número de participantes por cada categoría expuesta; \%: Porcentaje. } & $\begin{array}{c}44,8 \text { / } 60 \\
\text { Alta } \\
\end{array}$ \\
\hline
\end{tabular}

Fuente: la autora, procesamiento estadístico de los datos 
Tabla 3. Variables con asociaciones significativas con la Competencia para el cuidado

\begin{tabular}{|c|c|}
\hline $\begin{array}{c}\text { Dimensión de la } \\
\text { Competencia para el } \\
\text { cuidado }\end{array}$ & Variable significativa \\
\hline \multirow{2}{*}{ C- Conocimiento } & $\begin{array}{c}\text { Pulses del paciente (Chi-cuadrado de } \\
\text { Pearson - p: 0,004). }\end{array}$ \\
\hline & $\begin{array}{l}\text { Pulses del cuidador (Chi-cuadrado } \\
\text { de Pearson -p: 0,044). }\end{array}$ \\
\hline \multirow{5}{*}{$\begin{array}{l}\text { U- Unicidad } \\
\text { (condiciones personales) }\end{array}$} & $\begin{array}{l}\text { Pulses del paciente (Chi-cuadrado de } \\
\text { Pearson -p: 0,025). }\end{array}$ \\
\hline & $\begin{array}{l}\text { Escolaridad del cuidador (Chi-cua- } \\
\text { drado de Pearson -p: 0,022). }\end{array}$ \\
\hline & $\begin{array}{l}\text { Compromiso religioso del cuidador } \\
\text { (Chi-cuadrado de Pearson -p: 0,038). }\end{array}$ \\
\hline & $\begin{array}{c}\text { Carga percibida por el cuidador } \\
\text { (Chi-cuadrado de Pearson -p: 0,021). }\end{array}$ \\
\hline & $\begin{array}{c}\text { Edad del paciente (Prueba de la } \\
\text { Mediana - p: 0,017). }\end{array}$ \\
\hline \multirow{2}{*}{$\begin{array}{l}\text { I- Instrumental y } \\
\text { procedimental }\end{array}$} & $\begin{array}{l}\text { Compromiso religioso del cuidador } \\
\text { (Chi-cuadrado de Pearson - p: 0,037) }\end{array}$ \\
\hline & $\begin{array}{c}\text { Carga percibida por el cuidador } \\
\text { (Chi-cuadrado de Pearson -p: 0,031). }\end{array}$ \\
\hline \multirow{5}{*}{ D- Disfrutar (bienestar) } & $\begin{array}{c}\text { Pulses del paciente (Chi-cuadrado de } \\
\text { Pearson - p: 0,012). }\end{array}$ \\
\hline & $\begin{array}{c}\text { Género del paciente (Chi-cuadrado } \\
\text { de Pearson - p: 0,039). }\end{array}$ \\
\hline & $\begin{array}{c}\text { Género del cuidador (Chi-cuadrado } \\
\text { de Pearson - p: 0,049). }\end{array}$ \\
\hline & $\begin{array}{c}\text { Carga percibida por el paciente } \\
\text { (Chi-cuadrado de Pearson - p: 0,035) }\end{array}$ \\
\hline & $\begin{array}{l}\text { Compromiso religioso del cuidador } \\
\text { (Chi-cuadrado de Pearson - p: 0,033) }\end{array}$ \\
\hline
\end{tabular}

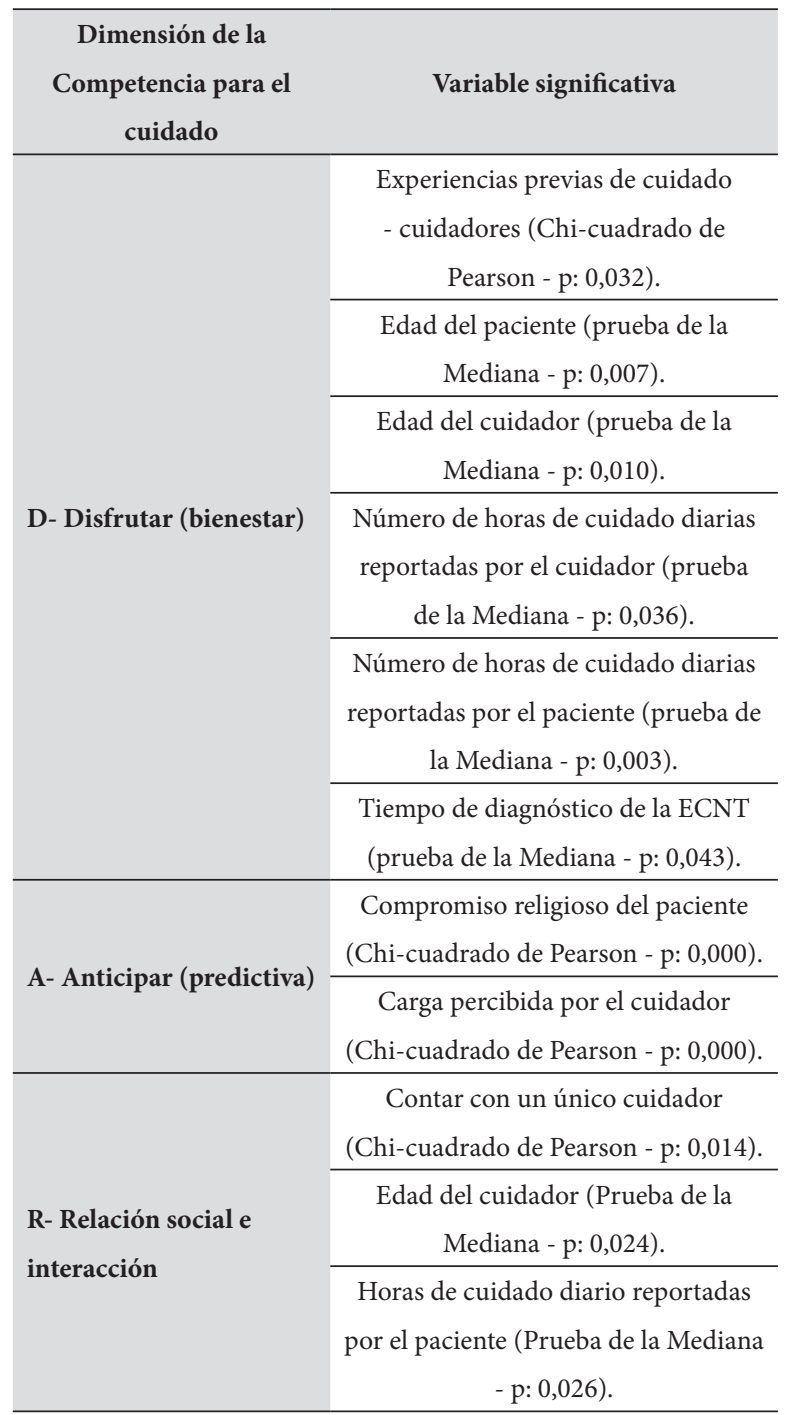

Fuente: la autora, procesamiento estadístico de los datos 


\section{DISCUSIÓN}

En el presente estudio, las díadas de cuidado mostraron características similares a otras ivestigaciones en la materia, entre estas:que los cuidadores en su mayoría son de género femenino, con edades por encima de los 40 años, con niveles educativos bajos y medios, con su ocupación definida como el estar en el hogar, son solteros o casados y son de estratos socioeconómicos bajos (12) (13) (14). Lo anterior muestra que la mujer en el rol de cuidadora es, en definitiva, un eje primordial del cuidado en el hogar. De igual manera, las personas receptoras de cuidado son, en su mayoría, de género femenino y tienen edades superiores a los 60 años, presentan un nivel bajo de escolaridad, pertenecen a estratos socioeconómicos bajos y tienen antecedentes patológicos de ECNT de alta frecuencia como son la HTA y la DM (15).

En relación con la percepción de carga y apoyo, se evidencia similitud en los hallazgos de este estudio frente a los resultados de otros en la temática, pues es frecuente que los cuidadores se perciban con sobrecarga, dadas sus responsabilidades y el tiempo de cuidado diario a sus familiares, lo que los hace más vulnerables a desarrollar el síndrome del cuidador y presentar múltiples afectaciones físicas y psicológicas (16-18); asimismo, las personas que reciben estos cuidados, en muchas ocasiones, se perciben a sí mismas como una carga para los demás. Sobre la satisfacción que las díadas de cuidado reciben, tanto a nivel psicológico como familiar, religioso, económico y social, de forma global, se puede decir que esta es percibida como mínima, situación que genera mayores riesgos en dicha población, pues el apoyo media el bienestar psicológico y promueve el bienestar en los cuidadores familiares (19) y, por tanto, también en las personas con ECNT que son cuidadas.

Frente a la autovaloración que las díadas de cuidado hacen del uso de los medios de comunicación e información, se evidenció que el acceso y uso del teléfono y la televisión reciben una percepción favorable, resultados que coinciden con lo encontrado en cuidadores familiares de personas con enfermedad respiratoria crónica (20). Este aspecto es de especial importancia en la gestión del cuidado, puesto que el uso de las tecnologías de la información y las comunicaciones optimiza muchos de los procesos requeridos al cuidar (21), ya que se trata de herramientas con un alto potencial para facilitar las actividades de cuidado de los cuidadores familiares, en la medida que permiten la comunicación continua y permanente y facilitan la transmisión de recomendaciones de cuidado y demás información valiosa para mejorar el nivel de competencia de cuidado.

Para hablar de la competencia de cuidado es necesario mencionar que esta incluye un potencial para cuidar y abarca la necesidad de conocer in- 
tegralmente a la persona que asumirá el cuidado en el hogar. De esta manera, el cuidador pasa a ser el principal actor del proceso, es por eso que Sánchez y Cols (2) hacen énfasis en la competencia para el cuidado en el hogar y sus dimensiones, como el conocimiento, la información y el reconocimiento de experiencias previas que inciden de forma directa en la carga del cuidado del cuidador.

Al evaluar los resultados de competencia para cuidar en el hogar, se obtuvo una valoración promedio alta por parte de los cuidadores potenciales, resultado que puede ser considerado positivo, dado que permite resaltar la percepción de seguridad en sus habilidades y preparación como personas cuidadoras. Estudios a nivel nacional, como el elaborado por Carrillo y cols (2015) evidencia que los cuidadores familiares presentan niveles más altos de competencia que las personas con enfermedad crónica $(22,23)$, aspecto que permite determinar la impotencia de su rol en las díadas de cuidado.

De igual manera, el nivel de competencia de cuidado en sus diferentes dimensiones se relacionó estadísticamente con aspectos como el nivel de dependencia de la persona cuidada, la escolaridad, la percepción de carga, el nivel de compromiso religioso, la edad de cuidador, el número de horas que esta al cuidado, y el tener experiencias previas en el cuidado. Lo anterior, se documenta como repuesta inicial a recomendaciones de es- tudios previos sobre conducir investigaciones que amplíen la relación entre sobrecarga y competencia de cuidado (24), siendo estas evidencias de importancia en la estructuración de intervenciones de enfermería para fortalecer la competencia de cuidado; la cual es un fenómeno central a ser intervenido durante la transición hospital-hogar y asegurar la continuidad del tratamiento (25).

Es necesario mencionar, como limitaciones de este estudio, que las asociaciones estadísticas mostradas llegan al nivel bivariado, lo que implica que no se controlaron factores de confusión. Se recomienda entonces realizar estudios con análisis multivariados en muestras más amplias, de las cuales se deriven la estructuración e implementación de intervenciones que fortalezcan la competencia de cuidado en esta población.

\section{CONFLICTOS DE INTERESES}

Los autores declaran no tener conflicto de interés.

\section{FINANCIACIÓN}

La financiación de este proyecto contó con el aporte de las instituciones participantes y de los investigadores. 


\section{AGRADECIMIENTOS}

Al Hospital Santa Clara y a la Universidad Nacional de Colombia, al equipo de trabajo de este estudio y demás personas que intervinieron en su realización.

\section{REFERENCIAS}

1. Carrillo-González GM, Barrera-Ortiz L, Chaparro-Díaz L, Pinto-Afanador N y SánchezHerrera B. Necesidades de cuidado en personas con cáncer durante la transición hospitalhogar. En: Grupo de Cuidado. El cuidado de enfermería y las metas del milenio - ISBN: 978958-761-355-1. 1 ra edición. Bogotá: Ediciones Universidad Nacional; 2012. 131-147.

2. Sánchez Herrera, B., Carrillo González, G. M., Barrera Ortiz, L., Chaparro Díaz, L. Carga del cuidado de la enfermedad crónica no transmisible. Aquíchan. 2013; 13 (2): 247-260

3. Pérez-Escoda N, Ceballos K, Ruiz-Bueno A. Competencias emocionales y depresión en cuidadores familiares de personas mayores dependientes. Ansiedad y Estrés; 2014: 20(2/3): 181-191.

4. Organización Mundial de la Salud. Enfermedades Crónicas y Promoción de la salud: Enfermedades crónicas principal causa de mortalidad. Consultado el 10 de marzo de 2015 Disponible en: http://www.who.int/chp/ chronic_disease_report/part1/es/index1.html

5. Acuña-Merchán L, Soler-Vanoy L, Sanchez $P$, Martínez-Ruiz D, Torres-Cortes L, Mercado $E$, et al. Situación de la Enfermedad Renal Crónica en Colombia 2013. Fondo Colombiano de Enfermedades de Alto Costo. Consultado el 07 de mayo de 2020. Disponible en: https://www.minsalud.gov.co/ sites/rid/Lists/BibliotecaDigital/RIDE/INEC/CAC/ SITUACION_DE_LA_ENFERMEDAD_RENAL_ CRONICA 2013.pdf

6. Carrillo-González GM, Barrera-Ortiz L, ChaparroDíaz L, Pinto-Afanador N y Sánchez-Herrera B. Cuidando a los cuidadores. Familiares de personas con enfermedad crónica ISBN: 978958-719-391-6. 1ra edición. Bogotá: Ediciones Universidad Nacional de Colombia; 2010. 360 p.

7. Barrera-Ortiz L, Pinto-Afanador N y SánchezHerrera B. "Estudio multicéntrico. Validación Programa cuidando a Cuidadores". Facultad de Enfermería, Universidad Nacional de Colombia. Archivo final de Investigación 2008.

8. Barrera L, Blanco L, Figueroa P, Pinto N. Habilidad de cuidadores familiares de personas con enfermedad crónica. Mirada internacional. Aquíchan; 2006; 6(1):22-33 
9. Aldana E.A., Barrera S.Y., Rodríguez K.A., Gómez O.J., Carrillo G.M. Competencia para el cuidado (CUIDAR) en el hogar de personas con enfermedad renal crónica en hemodiálisis. Enferm Nefrol. 2016; 19 (3): 265-273

10. Chaparro L, Sánchez B, Carrillo GM. Encuesta de caracterización del cuidado de la diada cuidador familiar - persona con enfermedad crónica. Rev. Cien. Ciudad. 2014; 11(2): 31-45.

11. Carrillo GM, Sánchez B, Arias EM. Validation of an instrument to assess the homecare competency of the family caregiver of a person with chronic disease. Investí Educ Enferm. 2015; 33(3): 449-455. http://dx.doi. org/10.17533/udea.iee.v33n3a08

12. Carreño S.P., Chaparro-Díaz L. Agrupaciones de cuidadores familiares en Colombia: perfil, habilidad de cuidado y sobrecarga. Pensamiento Psicológico, 2017, 15 (1), 87-101. http://dx.doi.org/10.11144/ Javerianacali.PPSI15-1.ACFC

13. Cardona D, Segura A, Berbesí D, Agudelo M. Prevalencia y factores asociados al síndrome de sobrecarga del cuidador primario de ancianos. Rev Fac Nac Salud Pública. 2013; 31: 30-9

14. Rodríguez-Albañil, Y., \& Quemba-Mesa, M. Nivel de sobrecarga en cuidadores informales de personas en situación de discapacidad cognitiva de Duitama. Estudio exploratorio. Revista Investigación en Salud Universidad de Boyacá, 2018, 5 (1), 86-98. https://doi.org/10.24267/23897325.270.

15. Benet RM, Morejón GA, Espinosa BAD, Landrove OO, Peraza AD, Orduñez GPO. Factores de riesgo para enfermedades crónicas en Cienfuegos, Cuba 2010. Resultados preliminares de CARMEN II. Finlay, 2017; 7 (1): $117-120$.

16. Flores N, Jenaro C, Moro L, Tomşa R. Salud y calidad de vida de cuidadores familiares $y$ profesionales de personas mayores dependientes: estudio comparativo. European Journal of investigation in Health, Psychology and Education. 2014, 4 (2): 79-88. https://doi. org/10.30552/ejihpe.v4i2.73

17. De Valle-Alonso M.J., Hernández-López I.E., Zúñiga-Vargas M.L., Martínez-Aguilera P. Sobrecarga y Burnout en cuidadores informales del adulto mayor. Enferm. Univ. 2015; 12 (1): 19-27

18. Zepeda-Álvarez P.J. y Muñoz-Mendoza C.L. Sobrecarga en cuidadores principales de adultos mayores con dependencia severa en atención primaria de salud. Gerokomos, 2019, 30 (1): 2-5. 
19. García F.E., Manquián E. y Rivas G. Bienestar psicológico, estrategias de afrontamiento y apoyo social en cuidadores informales. Psicop erspectivas, 2016, 15 (3): 101-111.

20. Pinzón EA, Carrillo GM. Carga del cuidado y calidad de vida en cuidadores familiares de personas con enfermedad respiratoria crónica. Rev. Fac. Nac. Salud Pública 2016; 34(2): 193201. http://dx.doi.org/10.17533/udea.rfnsp. v34n2a08

21. Vialart M.N. Information and communication technologies: a challenge for care management. Rev Cubana Enfermer. 2016; 32 (1): 118-125.

22. Nogueira M.A., Azeredo Z.A., Santos A.S. Competências do cuidador informal atribuídas pelos enfermeiros comunitários: um estudo Delphi. Rev. Eletr. Enf. 2012; 14(4): 749-759. https://doi.org/10.5216/ree.v14i4.13205

23. Carrillo-González G., Barreto-Osorio R., Arboleda L., Gutiérrez-Lesmes O., Melo B, Támara-Ortiz V. Competence for Home Health Care in Colombian People with Chronic Illness and their Caregivers. Rev. Fac. Med. 2015; 63 (4): 668-75. DOI: http://dx.doi.org/10.15446/ revfacmed.v63.n4.50322
24. Carreño-Moreno $\mathrm{S}$, Arias-Rojas $M$. Competencia para cuidar en el hogar y sobrecarga en el cuidador del niño con cáncer. Gaceta Mexicana de Oncología, 2016; 15 (6): 336-343. https://doi.org/10.1016/j. gamo.2016.09.009

25. Sánchez LM, Carrillo GM. Competencia para el cuidado en el hogar diada persona con cáncer en quimioterapia. Cuidador familiar. Psicooncología 2017;14: 137-48. https://doi. org/10.5209/PSIC.55817

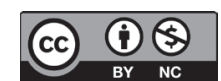

Esta obra está bajo una licencia de Creative Commons Reconocimiento-NoComercial 4.0 Internacional 